\title{
Trend and Policy of publishing open access article in China
}

\author{
HAIYAN LIU ${ }^{1}$ AND OLIVIER POURRET ${ }^{2}$
}

${ }^{1}$ East China University of Technology

${ }^{2}$ UniLaSalle

Presenting Author: hy.liu123@qq.com

Although Open Access (OA) publishing aims to make scientific achievements more accessible to readers, there is a current tendency that Chinese researchers prefer hybrid journals to OA ones when submitting Earth Sciences articles. Indeed, data obtained from the WoS categories "Geochemistry and Geophysics" and "Geology" and the Scopus category "Earth and Planetary Sciences" indicate that China has the lowest proportion (18-20\%) of OA articles published in 2018 among the six countries (USA, UK, France, Indonesia, South Africa). This is less than half of the highest proportion (46-54\%) possessed by UK. A historical national incentive encouraging researchers to publish articles in top journals (i.e. high JIF and first quartile) categorized by the Chinese Academy of Sciences is one of important causes for this trend. No need to pay APC for publishing research in a "high impact" predominantly English hybrid journal makes a contribution to the trend as well. However, the trend may change in response to a newly launched national-level policy in China in early 2020: to ban the use of journal-based metrics as assessment criteria for academic promotion and recruitment. Further, publishing in Chinese journals is being proposed as part of the prerequisites for application of top national awards. The policy will give priority to considering the innovation of one's research work and significance of representative achievements in solving practical problems. A move away from high JIF journals to Chinese journals could be a real game changer as Chinese researchers produce the bulk of articles, the majority of which have page charges and are fully OA by default. Specifically, the new policy tackles perverse incentives that drive the "publish or perish" culture which might be encouraging questionable research. 